\title{
A Novel Technique in Fingerprint Identification using Relaxation labelling and Gabor Filtering
}

\author{
T. Arumuga Maria Devi ${ }^{1}$, Mariadas Ronnie C.P ${ }^{2}$ \\ ${ }^{1}$ Assistant Professor, Centre for Information Technology and Engineering, Manonmaniam Sundaranar \\ University, Tirunelveli \\ ${ }^{2}$ P G Scholar, Centre for Information Technology and Engineering, Manonmaniam Sundaranar University, \\ Tirunelveli.
}

\begin{abstract}
This paper targets to reconstruct the original fingerprint image from minutiae information using relaxation labeling and Gabor filtering which can reveal substantial details such as the orientation field. The proposed technique utilizes minutiae information to estimate the orientation map of the parent fingerprint. First, the Gabor filter is used to extract the properties of the fingerprint image by using ridge mapping and thinning. Second, Relaxation Labeling uses the Gabor image to predict the orientation and direction of the image to generate the point care index image and singular point image specifications. Finally, the image properties is extracted to produce the minutiae information.
\end{abstract}

\section{Index Terms:- Orientation Map, Thinning, Gabor Filter, Relaxation Labeling, Minutiae.}

\section{INTRODUCTION}

The process of fingerprint identification is one of the most well known and publicized biometrics. Because of its uniqueness and consistency, it has been used or person identification for over a century and now is becoming automated due to the advancement in computing capabilities. Among the most accepted techniques used in fingerprinting, a new technique is here applied that combines both relaxation labelling and Gabor filtering for detection and classification of fingerprint images. Fingerprints possess two main types of features that are used for automatic fingerprint identification and verification: (i) Ridge and furrow structure that forms a special pattern in the central region of the fingerprint and (ii) Minutiae details associated with the local ridge and furrow structure. The human fingerprint is comprised of various types of ridge patterns, traditionally classified according to the decades-old Henry system: left loop, right loop, arch, whorl, and tented arch. Loops make up nearly $2 / 3$ of all fingerprints, whorls are nearly $1 / 3$, and perhaps $5-10 \%$ are arches. These classifications are relevant in many large-scale forensic applications, but are rarely used in biometric authentication.

Many types of minutiae exist, including dots (very small ridges), islands (ridges slightly longer than dots, occupying a middle space between two temporarily divergent ridges), ponds or lakes (empty spaces between two temporarily divergent ridges), spurs (a notch protruding from a ridge), bridges (small ridges joining two longer adjacent ridges), and crossovers (two ridges which cross each other). The classifications of the fingerprint are as shown in the figure:

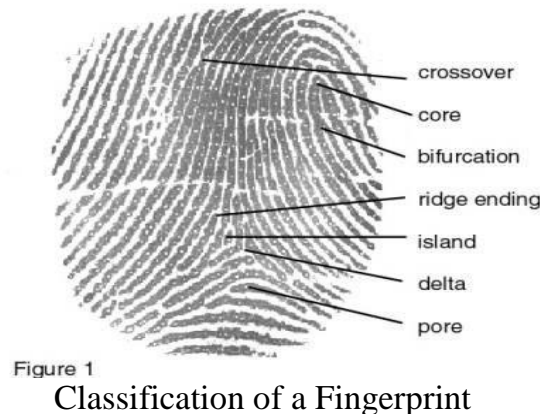

Fingerprint matching techniques can be placed into two categories: minutiae-based and correlation based. But the commonly used technique with minimum FAR and FRR is Minutiae-based techniques. In this process, the minutiae point is found first and then is mapped with their relative placement on the finger. However, there are some difficulties when using this approach. It is difficult to extract the minutiae points accurately when the fingerprint is of low quality. Also this method does not take into account the global pattern of ridges and furrows. Fingerprint Verification System is a system that determines the correspondence of an input fingerprint with a template fingerprint stored in data base. In a verification fingerprint system, the template 
fingerprint image is obtained in the enrollment phase. After that verification process takes place by a inputting the sample of the user's fingerprint at sensor. Such input fingerprint must be processed, in the preprocessing step. The preprocessing includes image enhancement, gray level adjust, ridge thinning, etc. After the fingerprint image has been preprocessed, the feature extraction block extracts the relevant information that will be used for matching with the template fingerprint. Finally a verification decision is made with the results or percentages of similarity obtained from the matching step.

Based on the theory of fingerprint classification, a single finger print of person is stored. This single print can be thumb print or print of index finger. One obvious advantage of this approach is that it will considerably reduce the amount of memory required to store the fingerprint template as only one print is stored instead of 10 prints for an individual. During the enrollment process, sensor senses the fingerprint, then next step is feature extraction, here minutiae points are extracted. After this step, a classifier is used to check the classification of input template that whether it is left-loop, right-loop, arch or whorl. After classification, the input template will be stored. In the verification process, here the finger or finger print is inputted and then its features are extracted and a final template is generated for matching. The template will perform match from this extracted domain. This process will be fast and more efficient than the conventional fingerprint identification systems.

\section{Discrete Wavelet Transform (DWT)}

Discrete Wavelet Transform (DWT) is the most popular transform for image-based application. They have lower computational complexity, and they provide interesting features such as component and resolution scalability and progressive transmission. A 2-dimensional wavelet transform is applied to the original image in order to decompose it into a series of filtered sub band images. At the top left of the image is a low-pass filtered version of the original and moving to the bottom right, each component contains progressively higher-frequency information that adds the detail of the image. It is clear that the higher-frequency components are relatively sparse, i.e., many of the coefficients in these components are zero or insignificant. The wavelet transform is thus an efficient way of decorrelating or concentrating the important information into a few significant coefficients.

To extract the orientation fields of the fingerprint image region, Local Fourier analysis method is used to estimate the initial orientation field. A fingerprint image $I(x ; y)$ is divided into blocks of $16 \times 16$ pixels. A 2D sine wave can approximate the ridge structure of a block and thus the task of estimating local ridge orientation is transformed by estimating the parameters of sine wave in each block. Centered at each block, the local image in the $64 \times 64$ window is multiplied by a bivariate Gaussian function $(s=16)$. The Discrete Fourier Transform (DFT), $F(u ; v)$, of the resulting image obtained is computed and the amplitude of low frequency components, that is, the points within 3 pixels from the center in the frequency domain is set to 0 . In the frequency domain, one or two local maximum points with the greatest amplitude are found. Each of these points corresponds to a 2D sine wave $\mathrm{w}(x ; y)=a \sin (2 \mathrm{p} f(\sin (\mathrm{q}) x+\cos (\mathrm{q}) y)+\mathrm{F})$, where $a, f, \mathrm{q}$, and $\mathrm{F}$ represent the amplitude, frequency, orientation, and phase, respectively.

\section{Relaxation Labeling}

Relaxation labeling (RL) [10] is originally referred as a heuristic dynamical system and it specifies a discrete time dynamical system in which class labels were refined while taking relationships in the pixel and label array into account. As the technique came upon interest and grew, many bifurcations, off shoots and generalizations of the basic idea developed. The examples are the product combination rule, the optimization approach, projected gradient descent, discrete relaxation, and probabilistic relaxation. RL in its basic form is a discrete time update equation that is suitably modified depending on the problem of interest which are Image matching, segmentation, or classification. The labeling strategies are encompassed by the term relaxation. The common aim is to improve the consistency of object classification. This is achieved by utilizing observational information in the form of measurements pertaining to objects and prior knowledge of the constraints which apply in a particular labeling application. The relaxation processes draw on this information in a number of distinct ways. For instance, discrete relaxation is concerned with the updating of object-labels so as to achieve global consistency. Probabilistic relaxation, on the other hand, is concerned with updating label probabilities using the evidence provided by neighboring objects. Both types of relaxation process have received considerable attention in the literature and have a variety of realizations. The original discrete relaxation algorithm was developed by Waltz. The constraint filtering algorithm, which is a method, can be used to obtain globally consistent label configurations. It shows how knowledge relating to a particular labeling application could be represented by a dictionary of symbolic constraints applying to groups of objects. Unfortunately, the constraint filtering algorithm had a number of shortcomings. It proved difficult to regulate for general labeling problems and by virtue of the purely symbolic representation of the labeling task, failed to admit the wealth of available observational information. Although the extensions of the relaxation concept admit observational information, 
they have not drawn on Waltz's dictionary idea to model the label process. The relaxation method has a vastly improved capacity to represent highly structured labeling applications.

\section{Algorithm: Relaxation Labeling Algorithm}

Initialization: set time t to zero to obtain initial label probabilities such as;

Let $\mathrm{P}(0)=(\mathrm{p} 1(0), \mathrm{p} 2(0) \ldots \mathrm{pn}(0))$

A label is then chosen from the random probabilities $\mathrm{p}_{\mathrm{i}}(\mathrm{t})$

while true do

for $i=1,2 \ldots n$ do

//To select a label randomly

end

for $i=1,2 \ldots n$ do

Let $\mathrm{q}$ be the label selected for the object $\mathrm{o}_{\mathrm{i}}$

Compute the response $\beta_{\mathrm{iq}}$ to $\mathrm{O}_{\mathrm{i}}$ as

$$
\beta_{\mathrm{iq}}=(1 / \mathrm{N})_{\mathrm{ij}}\left(\mathrm{q}, \mathrm{s}_{\mathrm{j}}\right)
$$

Where $\mathrm{s}_{\mathrm{j}}$ is the label selected for object $\mathrm{o}_{\mathrm{j}}, \mathrm{q}$ is the label selected for the object $\mathrm{o}_{\mathrm{i}}$

end

for $i=1,2 \ldots n$ do

Let $\mathrm{q}$ be the label selected for object $\mathrm{o}_{\mathrm{i}}$

$\mathrm{p}_{\mathrm{i}}(\mathrm{t})$ is updated as

$$
\begin{aligned}
& \mathrm{p}_{\mathrm{iq}}(t+1)=\mathrm{p}_{\mathrm{iq}}(t)+\alpha \beta_{\mathrm{iq}}\left(1-\mathrm{p}_{\mathrm{iq}}(t)\right) \\
& \mathrm{p}_{\mathrm{ir}}(t+1)=\mathrm{p}_{\mathrm{ir}}(t)+\alpha \beta_{\mathrm{iq}} \mathrm{p}_{\mathrm{ir}}(t), \mathrm{r} \neq \mathrm{q}
\end{aligned}
$$

end

if probability vectors have converged then

break.

end

else

$t=t+1$

end

end

\section{Minutiae Extraction}

The fingerprint image that is inputted is the gray scale image of a person, which has intensity values ranging from 0 to 255 . In a fingerprint image, the ridges appear as dark lines while the valleys are the light areas between the ridges. Minutiae points are the locations where a ridge becomes discontinuous. A ridge can either 
come to an end, which is called as termination or it can split into two ridges, which is called as bifurcation. Binarization is used to convert gray scale image into binary image by fixing the threshold value. The pixel values above and below the threshold are set to ' 1 ' and ' 0 ' respectively. The binarized image is thinned using Gabor Filter to reduce the thickness of all ridge lines to a single pixel width to extract minutiae points effectively. Thinning does not change the location and orientation of minutiae points compared to original fingerprint which ensures accurate estimation of minutiae points. Thinning preserves outermost pixels by placing white pixels at the boundary of the image, and as a result first five and last five rows, first five and last five columns are assigned value of one. Dilation and erosion are used to thin the ridges. The minutiae location and the minutiae angles are derived after minutiae extraction. To calculate the bifurcation angle, the advantage of the fact that termination and bifurcation are dual in nature is used. The termination in an image corresponds to the bifurcation in its negative image. Hence by applying the same set of rules to the negative image, the bifurcation angles are obtained. To compare the input fingerprint data with the template data, Minutiae matching is used. During the matching process, each input minutiae point is compared with template minutiae point. In each case, template and input minutiae are selected as reference points for their respective data sets. The reference points are used to convert the remaining data points to polar coordinates.

\section{TECHNIQUE}

The problem areas are divided into,

1. Pre-processing the test Fingerprint.

2. Extract the minutiae points.

3. Matching test Fingerprint. .

To handle the problem of fingerprint identification, first the fingerprint image is gray scaled. Using the methods, the orientation of the image and the frequency of the image is estimated. Then the quality of the image is estimated using dilation and erosion and thinning is performed on the image. Ridge mapping is performed and Gabor filter is used to obtain the features of the image. The Gabor filtered image is converted to a binary image and vectorization is performed to find the direction of the fingerprint. By using the technique of relaxation labelling, labelling is performed on the image. Finally, the minutiae property is obtained which shows better result.

\section{FIGURES}

\section{Gabor Filtered Outputs}

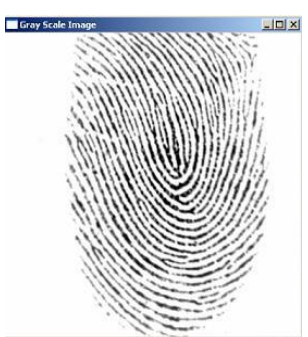

Figure 2: Gray Scale Image

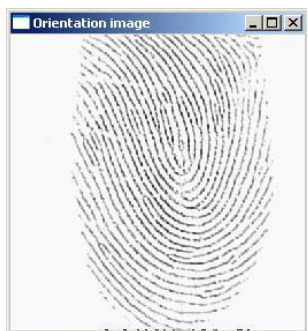

Figure 4: Orientation Image

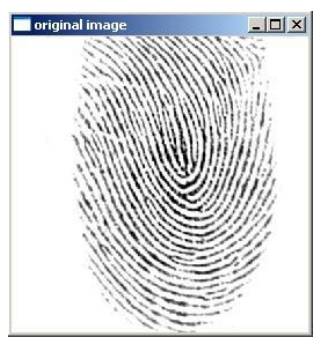

Figure 3: Original Image

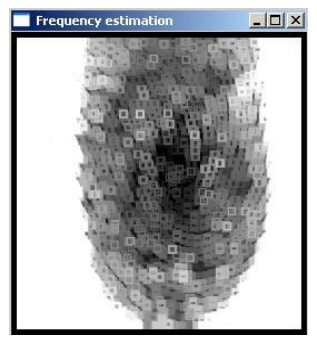

Figure 5: Frequency Estimation 


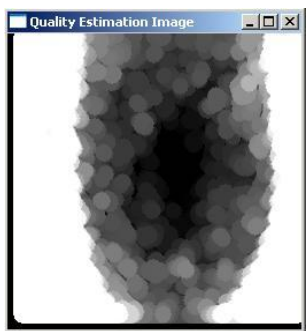

Figure 6: Quality Estimation

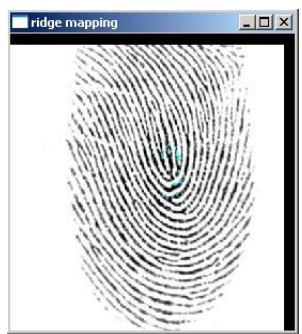

Figure 8: Ridge Mapping

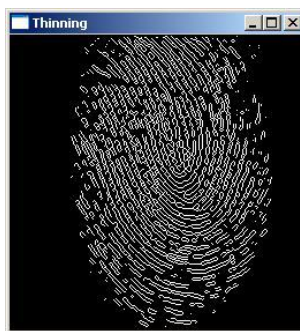

Figure 7: Thinning Image

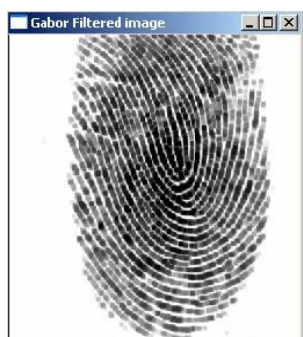

Figure 9: Gabor Filtered Image

Relaxation Labelling Outputs

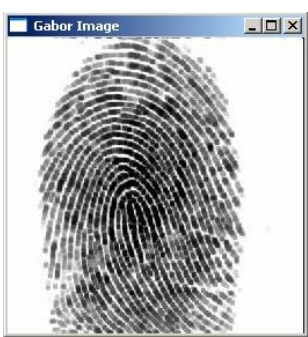

Figure 10: Gabor Image

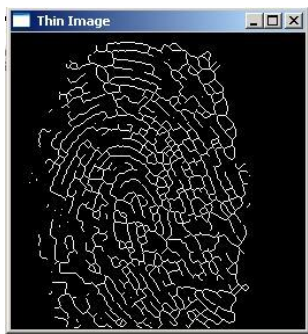

Figure 12: Thin Image

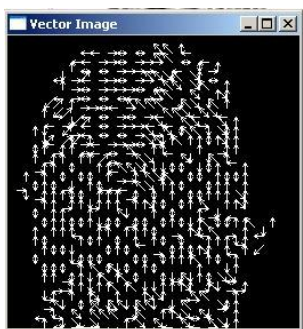

Figure 14: Vector Image

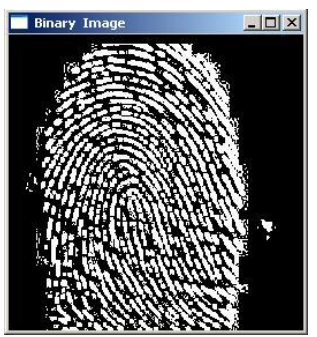

Figure 11: Binary Image

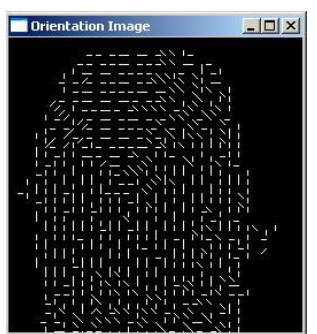

Figure 13: Orientation Image

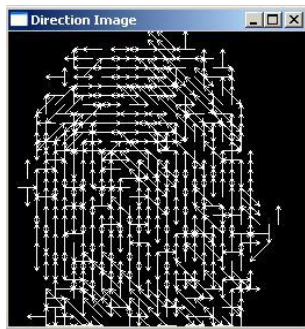

Figure 15: Direction Image 


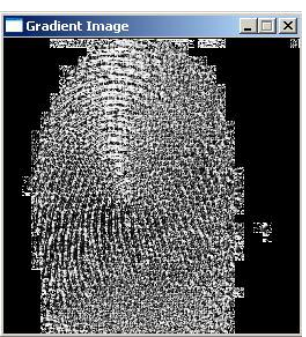

Figure 16: Gradient Image

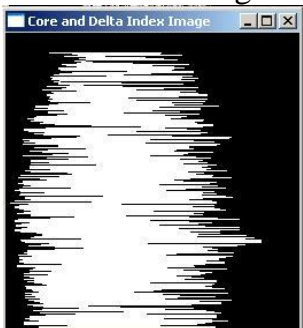

Figure 18: Core and Delta Index Image

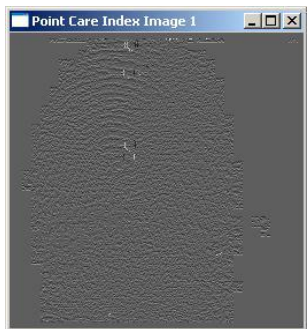

Figure 20: Point Care Index Image specific

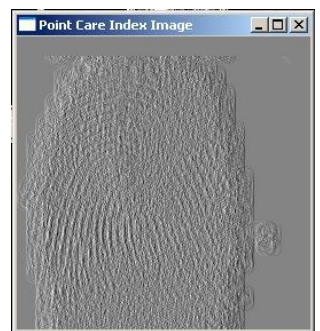

Figure 17: Point Care Index Image

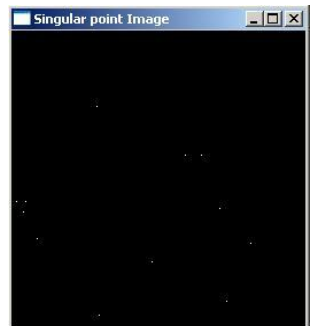

Figure 19: Singular Point Image

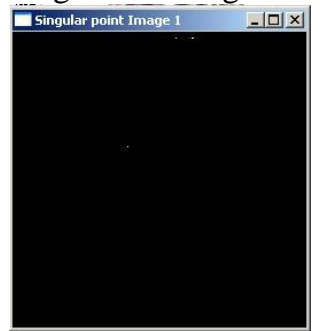

Figure 21: Singular Point Image specific

\section{Extraction Outputs}

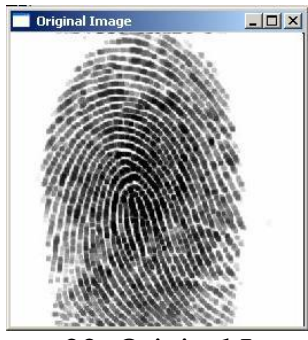

Figure 22: Original Image

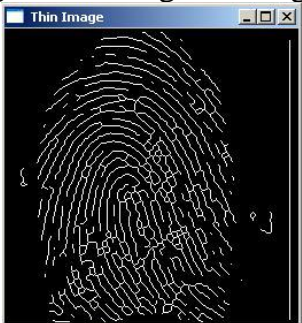

Figure 24: Thin Image

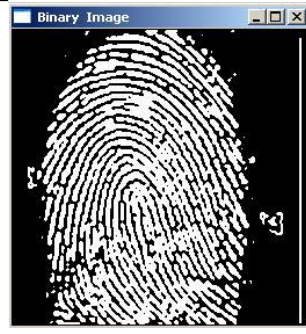

Figure 23: Binary Image

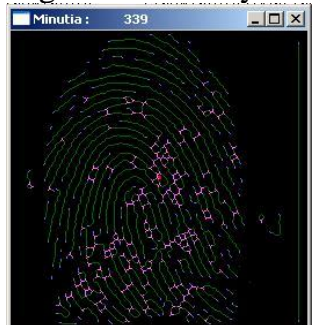

Figure 25: Minutiae

\section{CONCLUSIONS}

In this paper, we presented Fingerprint matching using Relaxation labeling and Gabor filtering. The pre-processing of the original fingerprint involves image binarization, ridge thinning, and noise removal. Fingerprint Recognition using Minutia Score Matching method is used for matching the minutia points. The proposed method gives better values compared to the existing method.

\section{ACKNOWLEDGMENT}

The authors would like to thank the members of the Dept. of CITE, M S University, Tirunelveli for various algorithms used in this research, and all the people who helped in preparing and carrying out the experiments and data collection. 


\section{REFERENCES}

1). D. Maltoni, D. Maio, A. K. Jain, and S. Prabhakar, Handbook of Fingerprint Recognition (Second Edition). Springer, 2009.

2). A. K. Jain and J. Feng, "Latent palmprint matching," IEEE Transactions on Pattern Analysis and Machine Intelligence, vol. 31, no. 6, pp. 1032-1047, 2009.

3). Y. Wang, J. Hu, and D. Phillips, "A fingerprint orientation model based on 2D Fourier expansion (FOMFE) and its application to singular-point detection and fingerprint indexing," IEEE Transactions onPattern Analysis and Machine Intelligence, vol. 29, no. 4, pp. 573- 585, 2007.

4). ] L. Hong, Y. Wan, and A. K. Jain, "Fingerprint image enhancement: Algorithm and performance evaluation," IEEE Trans. Pattern Anal.Mach. Intell., vol. 20, no. 8, pp. 777-789, Aug. 1998.

5). A. K. Jain and J. Feng, "Latent fingerprint matching," IEEETrans. Pattern Anal. Mach. Intell., vol. 33, no. 1, pp. 88-100, Jan. 2011.

6). H. Tang, W. Lu, C. Che, and K. Ng, "Gold nano particles and imaging mass spectrometry: Double imaging of latent fingerprints," Anal.Chem., vol. 82, no. 5, pp. 1589-1593, 2010.

7). X. Fan, D. Liang, and L. Zhao, "A scheme for separating overlapped fingerprints based on partition mask," (in Chinese) Comput. Eng.Applicat., vol. 40, no. 2, pp. 80-81, 2004.

8). A. K. Jain and J. Feng, "Latent palmprint matching," IEEE Trans.Pattern Anal. Mach. Intell., vol. 31, no. 6, pp. 1032-1047, Jun. 2009.

9). M. Pelillo, F. Abbattista, and A. Maffione, "Evolutionary learning for relaxation labeling processes," in Proc. $A I^{*} I A, 1993$, pp. 230-241.

10). M. Pelillo and M. Refice, "Learning compatibility coefficients for relaxation labeling processes," IEEE Trans. Pattern Anal. Mach. Intell., vol. 16, no. 9, pp. 933-945, Sep. 1994.

11). L. Padró, "Pos tagging using relaxation labelling," in Proc. Int. Conf. Computational Linguistics(COLING), 1996, pp. 877-882.

12). J. Kittler and J. Illingworth, "Relaxation labelling algorithms - A review," Image Vision Comput., vol. 3, no. 4, pp. 206-216, 1985.

13). A. J. Stoddart, M. Petrou, and J. Kittler, "On the foundations of probabilistic relaxation with product support," J. Math. Imag. Vision, vol. 9, no. 1, pp. 29-48, 1998.

14). A. Rangarajan, "Self-annealing and self-annihilation: Unifying deterministic annealing and relaxation labeling," Pattern Recognit., vol. 33, no. 4, pp. 635-649, 2000.

15). A. Rosenfeld, R. A. Hummel, and S. W. Zucker, "Scene labeling by relaxation operations," IEEE Trans. Syst., Man, Cybern., vol. 6, no. 6, pp. 420-433, Jun. 1976.

16). M. Thathachar and P. Sastry, "Relaxation labeling with learning automata," IEEE Trans. Pattern Anal. Mach. Intell., vol. 8, no.2, pp. 256-268, Feb. 1986.

17). J. Daugman, "Uncertainty relation for resolution in space, spatial frequency, and orientation optimized by two-dimensional visual cortical filters," J. Opt. Soc. Amer. A, vol. 2, no. 7, pp. 1160-1169, 1985.

18). J. Feng, A. K. Jain, and A. Ross, "Detecting altered fingerprints,"in Proc. 20th Int. Conf. Pattern Recognition (ICPR), 2010, pp.1622-1625

19). J. Zhou and J. Gu, "A model-based method for the computation of fingerprints' orientation field," IEEE Transactions on Image Processing, vol. 13, no. 6, pp. 821-835, 2004.

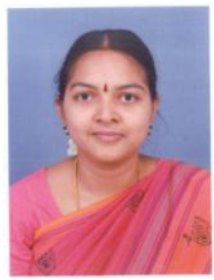

\section{AUTHORS}

T. Arumuga Maria Devi received B.E. Degree in Electronic and Communication Engineering from Manonmaniam Sundaranar University, Tirunelveli India in 2003, M.Tech degree in Computer and Information Technology from Manonmaniam Sundaranar University, Tirunelveli, India in 2005. Currently, she is doing Ph.D in Computer and Information Technology and also the Assistant Professor of Centre for Information Technology and Engineering of Manonmaniam Sundaranar University. Her research interests include Signal and Image Processing, Multimedia and Remote Communication.

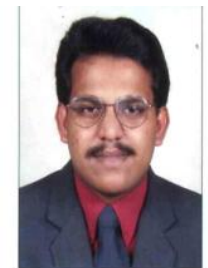

Mariadas Ronnie C.P received M.Tech in Computer and Information Technology (CIT) from Manonmaniam Sundaranar University, Tirunelveli, India in 2011, completed M.Phil degree in Information Technology (IT) at Manonmaniam Sundaranar University in the year 2012, received MCA Degree from Bharathiar University, Coimbatore, India in 2001. His research interest includes Digital Image Processing, Artificial Intelligence and Data Mining. 